\title{
Strictures, rings, webs and spasm
}

\author{
D. A. W. EDWARDs* \\ M.A., M.D., F.R.C.P. \\ Medical Research Council Gastroenterology Unit, Central Middlesex Hospital, and
Department of Clinical Research*
}

\begin{abstract}
Summary
The pathology of benign obstructing lesions is presented together with the mechanical problems they create, their radiological appearance, the symptoms which arise from them, and their differential diagnosis by symptoms, based on a study of 230 obstructing lesions.
\end{abstract}

\section{Spasm}

Spasm is mentioned only to discount it. Spasm is a nebulous concept of muscle contracting in response to unspecified irritation, or local inflammation, or to abnormal neuronal impulses. There is no radiographic nor manometric evidence that this happens. Increased resistance to stretch in the region of inflammation seems likely to result from the changes in the consistency of the tissue because the muscle in the wall contracts and relaxes normally but the zone will not distend with a bolus. What is called cricopharyngeal spasm is usually an extra-mucosal tissue change which has nothing to do with the cricopharyngeus. Sudden chest pain of oesophageal pain distribution without visible or demonstrable cause is not uncommon but there is no evidence, only presumption, for considering it is caused by muscle spasm, and the explanation is simply unknown. The subject can drink copiously during the pain and, if bolus obstruction to food is present, careful radiological examination has always demonstrated a ring stricture in my own experience.

\section{Webs}

Pharyngo-oesophageal webs occur within a few centimetres of the distal end of the pharynx, are covered on both sides with normal looking stratified squamous epithelium with a subepithelial layer of connective tissue and a few round cells. There is no good evidence of inflammatory tissue. They may be single but are often multiple. Equally common are constrictions or strictures which look as though a piece of thread or a sleeve of rigid tissue had been tied round the lumen. The sleeves may be up to $1 \mathrm{~cm}$ long and are usually single but the ring constrictions are

* Medical Research Council Department of Clinical Research, University College Hospital Medical School, London. often multiple and may reduce the lumen to 2 or 3 $\mathrm{mm}$. The epithelium is normal and the lesion is an inflammatory one of unknown aetiology in the subepithelial tissue.

The radiographic appearance is best seen by slow motion cinematography because the rate of movement of the wall and the contents is that of the speed of striated muscle. The patient drinks in the head down position, supine with the head turned to one side, because the distal constrictions are better visualized in the lateral view when gravity holds the barium in the upper oesophagus.

The symptom pattern is characteristic. Bolus obstruction to solids according to their size and consistency develops and progresses slowly over years. The impact is not painful but can be alarming because it may induce a feeling of choking, and occurs within $1 \mathrm{sec}$ of the swallow movement. The food may leave the mouth but is felt in the back of the pharynx and can be returned to the mouth without regurgitation. Liquids can be drunk at normal speed and without inducing coughing until the lumen becomes very narrow and only liquidized food is possible. When the constriction is severe each mouthful has to be swallowed slowly and requires firm pressure to force it through the narrow opening, often creating a loud noise as it does so.

\section{Rings}

Ring strictures (sometimes called Schatzki rings, contraction rings) form by an increased resistance to stretch at the junction of the squamous and columnar epithelium which most commonly occurs within the cardiac sphincter segment but may be proximal to it. Occasionally they appear to lie within the stomach The histological detail has been described infrequently, and some rings appear not to contain any inflammatory tissue, others do. The cause is unknown but almost all ring strictures are associated with the capacity to herniate some stomach and with radiographic reflux.

The radiographic demonstration requires very careful attention to technique and many ring strictures are frequently missed. The oesophagus proximal and distal to the ring must be well distended in order to demonstrate the ring of resistance to 
stretch and this is best achieved if the patient drinks barium continuously in the supine $10^{\circ}$ head-down position with the right side slightly raised. A large bolus of barium-impregnated bread or a barium marshmallow may be necessary to demonstrate the obstruction. If the relevant symptoms are present the patient should be believed and the radiologist should persevere. The ring appears as a constriction of less than $12 \mathrm{~mm}$ diameter and 2-3 mm thick.

Symptoms correlate well with the histological and radiographic appearance. Bolus obstruction occurs at the ring with the dramatic onset of pain or discomfort and a sense of obstruction occurs, sporadically at first, and later is more obviously related to the size and consistency of a bolus. Sometimes the bolus passes through, often it has to be regurgitated. There may be no progression for years and only a few episodes a year which seem most likely to occur when eating in company. The oesophageal epithelium is not hypersensitive to acid or hot or alcoholic drinks and there is no pain except when solids stick. There may or may not be heartburn.

These rings are missed by the endoscopist even more often than by the radiologist. The direction and amount of force applied in distending the wall during eating and drinking can be simulated by liquid or bread-soaked barium but are very different from the direction and amount of force at the end of an endoscope which usually temporarily stretches the ring which flips back into place when the endoscope is withdrawn. The most certain way of confirming the ring is to push a finger up the oesophagus through a gastrotomy and feel the edge of the squamocolumnar-junction.

\section{Peptic strictures}

The most important feature of the pathology of strictures is the stage of no return. Once a stricture has reached the irreversible stage only resection will give relief from socially and nutritionally destructive dysphagia, and resection will be successful in the long term in only a few. Irreversible stricture is a disaster which bougienage only slightly ameliorates. It is extremely rare for medical management to relieve or cure a stricture once it has developed and only surgery offers a hope of stopping an otherwise inexorable process. Patients with reflux symptoms. and evidence of oesophagitis who develop dysphagias for solids should have an urgent repair of the anti-o reflux mechanism.

The symptoms of stricture are characterized by painful obstruction to solids but not to liquids. Theळ strictured oesophagus behaves like an open tube with a rigidly narrowed zone against which a bolus? impacts depending on its size and consistency? Because there is no obstruction to fluid, drinking is $\overrightarrow{-}$ normal unless food has impacted, and the oesophagus does not dilate. Regurgitation follows foods impaction but the volume is small and never tastes] foul or of vomit. Pain is only present during their period of impaction and eases quickly as the bolus moves through the stricture or is regurgitated o Because the inflammatory process starts at the squamo-columnar junction and extends rapidly $\vec{\omega}$ around the circumference but progresses slowly, bolus obstruction occurs infrequently at first and the disability may progress over months and years. It is most unusual for the lumen to be reduced belowe. $4 \mathrm{~mm}$ so that even with a severe peptic stricture it is $\overrightarrow{0}$ possible to drink quickly. The time interval between the moment of swallowing and the sensation impact is a useful guide to the level of the strictupeo If the interval is 1-2 sec the stricture is probably? the upper third, if 2-3 sec in the middle third, and if more than $4 \mathrm{sec}$, in the lower third. The majority are in the sphincter segment which can contract too obliterate the lumen but cannot open fully because $\vec{\delta}$ of the tissue change in the wall.

The radiographic demonstration is easy if the patient drinks barium continuously when supine in the $10^{\circ}$ head-down position with the right sides slightly raised. The stricture may be missed when the patient is drinking barium erect. Loss of distensibility of the wall can only be demonstrated by fulf. distension proximal and distal to the stricture but iô is possible to demonstrate accurately the length, maximum bore, nature and toughness of a stricture and its proximal and distal shoulders by careful attention to technique. 